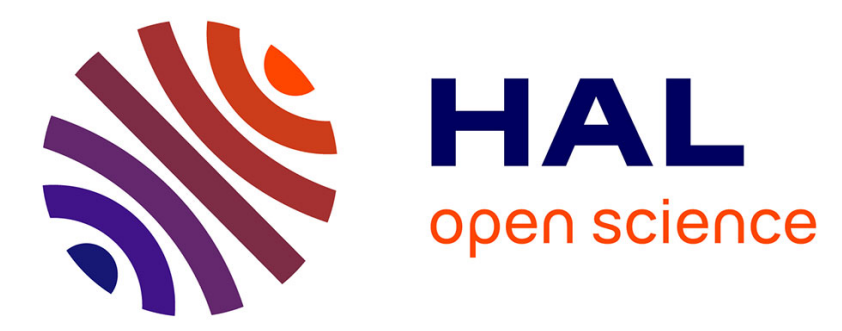

\title{
Isolated versus Coordinated Ramp metering: Field Evaluation Results of Travel Time Reliability and Traffic Impact
}

\author{
Neila Bhouri, Habib Haj Salem, Jari Kauppila
}

\section{- To cite this version:}

Neila Bhouri, Habib Haj Salem, Jari Kauppila. Isolated versus Coordinated Ramp metering: Field Evaluation Results of Travel Time Reliability and Traffic Impact. Transportation research. Part C, Emerging technologies, 2013, 28, pp.155-167. 10.1016/j.trc.2011.11.001 . hal-01272078

\section{HAL Id: hal-01272078 \\ https://hal.science/hal-01272078}

Submitted on 10 Feb 2016

HAL is a multi-disciplinary open access archive for the deposit and dissemination of scientific research documents, whether they are published or not. The documents may come from teaching and research institutions in France or abroad, or from public or private research centers.
L'archive ouverte pluridisciplinaire HAL, est destinée au dépôt et à la diffusion de documents scientifiques de niveau recherche, publiés ou non, émanant des établissements d'enseignement et de recherche français ou étrangers, des laboratoires publics ou privés. 


\title{
Isolated vs. Coordinated Ramp metering: Field Evaluation Results of Travel Time Reliability and Traffic Impact
}

\author{
Bhouri Neila $^{a}$, Haj-Salem Habib ${ }^{\mathrm{a}}$, Kauppila Jari ${ }^{\mathrm{b}}$
}

\begin{abstract}
:
Majority of management strategies today are still introduced for reducing congestion and improving safety. As a result, the evaluation of their impacts usually focuses on congestionrelated indicators, such as total time spent, total travel distance or mean speed. However, with the growing prosperity, consumers demand higher quality transport services, for which reliable transport networks are central. This paper is focused on the evaluation of the ramp metering impact based on both indices: traffic and the travel time reliability on the A6W motorway in Paris. Three strategies are implemented, tested and evaluated. Evaluations include more traditional traffic impacts on one hand and the travel time reliability evaluation on the other which corresponds to the add value of this paper. The results obtained indicate both improvement of the travel time reliability and the average travel time.
\end{abstract}

KEYWORDS: Travel time reliability, travel time, ramp metering, field test, feedback control, ALINEA strategy, coordinated strategy.

\footnotetext{
${ }^{a}$ Université Paris-Est, IFSTTAR, GRETTIA, F-93160, Noisy-le-Grand, France

(E-mail : neila.bhouri@ifsttar.fr, habib.haj-salem@ifsttar.fr)

${ }^{\mathrm{b}}$ Research Centre of the International Transport Forum at the OECD, 2 rue André Pascal, F-75775 Paris Cedex 16, France (E-mail: jari.kauppila@oecd.org).
} 


\section{INTRODUCTION}

Urban and freeway networks around major metropolitan areas have reached a high level of congestion. Congestion has not only impact on average travel time but also on travel time reliability (OECD/ECMT, 2007). There is much evidence that the variability of travel times may be more important than the average travel speed in that users of the network can plan their travel accordingly if a road is constantly congested while unpredictable travel conditions impose the greatest frustration (FHWA, 2006; McKinnon \& al., 2009; OECD/ITF, 2010; ). Indeed, drivers are used to the recurrent, everyday congestion. They don't like it, but they have to allow time for it. In order to ensure for ontime arrival, drivers often build in a buffer by leaving home early enough to get to their destination ontime. However, a more serious problem may arise when the level of congestion varies daily, making it difficult to predict the travel time. Reducing this uncertainty or unreliability is the main focus of this paper as travelers require consistency in their travel time.

A number of countries are looking at ways of improving the reliability of travel time while reliability has become increasingly important part of national transport policies. A wide range of policy instruments are available to improve reliability of transport and they can be distilled into four main options (OECD/ITF, 2010):

- Increasing capacity of infrastructure, either by supplying extra capacity or improving quality of existing one.

- Better management of existing capacity.

- Charging directly for reliability.

- Providing information to users mitigating the adverse effects of poor reliability.

It is acknowledged that many of the policy options mentioned above are already in use as congestion mitigation policies. Indeed, growing traffic leads to increasing severity, spatial extension and duration of congestion with two immediate consequences. One is that travel time increases on average. Another is that travel times become increasingly variable and unpredictable. Reliability is related to this variability in travel time. As congestion has negative impacts on reliability, one can expect that actions aimed at alleviating congestion can also improve the reliability of the transport network. However, it has been shown that reliability is not necessary same as congestion (OECD/ITF, 2010). It is therefore 
important to evaluate impacts of any remedial action separately both for average travel time (congestion) and for variability of that travel time (reliability).

This paper studies the reliability impact of implementing ramp metering on A6W motorway in Paris, France. Original field trials have been performed in the European research project EURAMP (EURAMP, 2006). Measurements of traffic volume, occupancy rate and speed as well as estimated travel time are used in the evaluation.

Ramp metering is a specific active management measure which employs traffic lights at the freeway on-ramps to control the traffic flow entering the motorway mainstream. It consists of limiting, regulating and timing the entrance of vehicles from one or more ramps onto the main line. As with many other highway policy strategies, ramp metering was originally designed to mitigate congestion impacts. It is recognized as the most direct and efficient way to control and upgrade motorway traffic, and a number of field tests of ramp control strategies in different countries are available showing the benefits to average travel time (Papageorgiou, et. al, 1991).

Until recently, assessments of management strategies have not included any quantified assessment of reliability benefits. In the recent paper, (Bhouri and Kauppila, 2011), impact of ramp metering on variability of travel time is presented using the isolated ramp metering strategy. This paper builds on that recent work and extends it by comparing two different freeway ramp metering strategies and presents their impact on reliability and travel time. The ramp metering techniques considered include the well known local ramp metering strategy, ALINEA (Asservissement Linéaire d'entrée sur Autoroute) (Haj-Salem et al, 1990, 1995, Papageorgiou et. al, 1991) and a coordinated startegy. ALINEA maintains locally the density on the carriage way around the critical value. Nevertheless, due to the synergetic effect of all metered on-ramps (they interact on each other at different time scale) a coordinated strategy could be more efficient than a local strategy. Therefore, we also present impacts of a new coordinated strategy, which we call CORDIN, on travel time and travel time reliability.

This paper is organized as follows: Section 2 briefly describes the used indices for the traffic impact assessment of any management strategies. Section 3 is dedicated to the description of the travel time reliability approaches and in particular the introduction of the definitions of a number of reliability indices used. Descriptions of applied ramp metering strategies on the test site are explained in detail in Section 4. Section 5 describes the test site and data used for the impact assessment. Section 6 summarizes results from the traditional traffic impact 
assessment and reliability assessment. Finally, Section 7 draws the main conclusions of this paper.

\section{DEFINITION OF THE TRAFFIC INDICES}

Among the management strategies, ramp metering has been introduced mainly for reducing congestion and improving safety and the evaluation of impacts usually focuses on congestion related indicators, such as average travel time, duration of congestion, mean speed, fuel consumption and emissions and risk index (Haj-Salem and Papageorgiou, 1995; Haj-Salem \& al., 2006, 2010). However, because the focus of this paper is in the measurement of the reliability impact, we only consider the impact of ramp metering on total time spent (TTS), total travel distance (TTD), mean speed (MS) and travel time (TT), which are closely linked (but not the same) to variability of travel time. The following summarises definitions for different traffic indices.

Total Time Spent (TTS) expressed in vehicles times hours (vh*h) is defined as:

$$
T T S=T \sum_{k=1}^{K} \sum_{i=1}^{N} \rho_{i}(k) \Delta_{i}
$$

where $\square$ measures the density of the segment $i, \quad T$ is for the real data collection time slice, $\square$ is the distance between two measured stations (i-1) and (i), $N$ is the number of the measurement stations and $K$ is the time horizon.

Total Travel Distance (TTD) expressed in vehicles times kilometers (vh*km), in turn, is defined as:

$$
T T D=T \sum_{k=1}^{K} \sum_{i=1}^{N} Q_{i}(k) \Delta_{i}
$$

Where $Q_{i}$ is the measured traffic volume of the station $i$.

Mean Speed (MS) is expressed in kilometers per hour $(\mathrm{km} / \mathrm{h})$ and is defined and TTD/TTS. 
Measurement of Travel Time is generally performed using a campaign of floating car surveys. These campaigns are, however, very expensive and could be replaced (when the site is fully equipped) by the use of the floating car algorithm. Moreover, several definitions of the travel time exits such as instantaneous travel time which is extensively used in France for the collective user information system (travel time display); generalized travel time which is defined as: $L / M S$ where $L$ is the total length of the motorway path; weighed travel time; and predicted travel time (DACCORD, 1998), (Van Lint \& Van der Zijpp, 2003).

In this paper, the travel time calculation was based on the application of the "floating car" algorithm using the real speed measurements of consecutive measurement stations on the motorway axis. The "floating car" algorithm consists of propagating (in space and time) a "fictive vehicle" for each time interval $(\Delta t)$ of the real data collection (see Figure 1 ). The position of the vehicle is updated each $\Delta$ t using the speed measurements of the next measurement station. Consequently, as soon as the time interval of the real data collection is lower, higher the accuracy of the travel time estimation becomes. In our case, the time interval is equal to $6 \mathrm{~min}$ and the mean distance between two consecutive measurement stations is around $500 \mathrm{~m}$. In order to taking into account the higher time interval, the "fictive vehicle” is propagated using several speed measurement of the consecutive stations during the same time interval $(\Delta t=6 \mathrm{~min})$. This approach tries to minimise the predicted travel time estimation errors. The travel time index will be used extensively for the reliability evaluation as reported in the next section. 


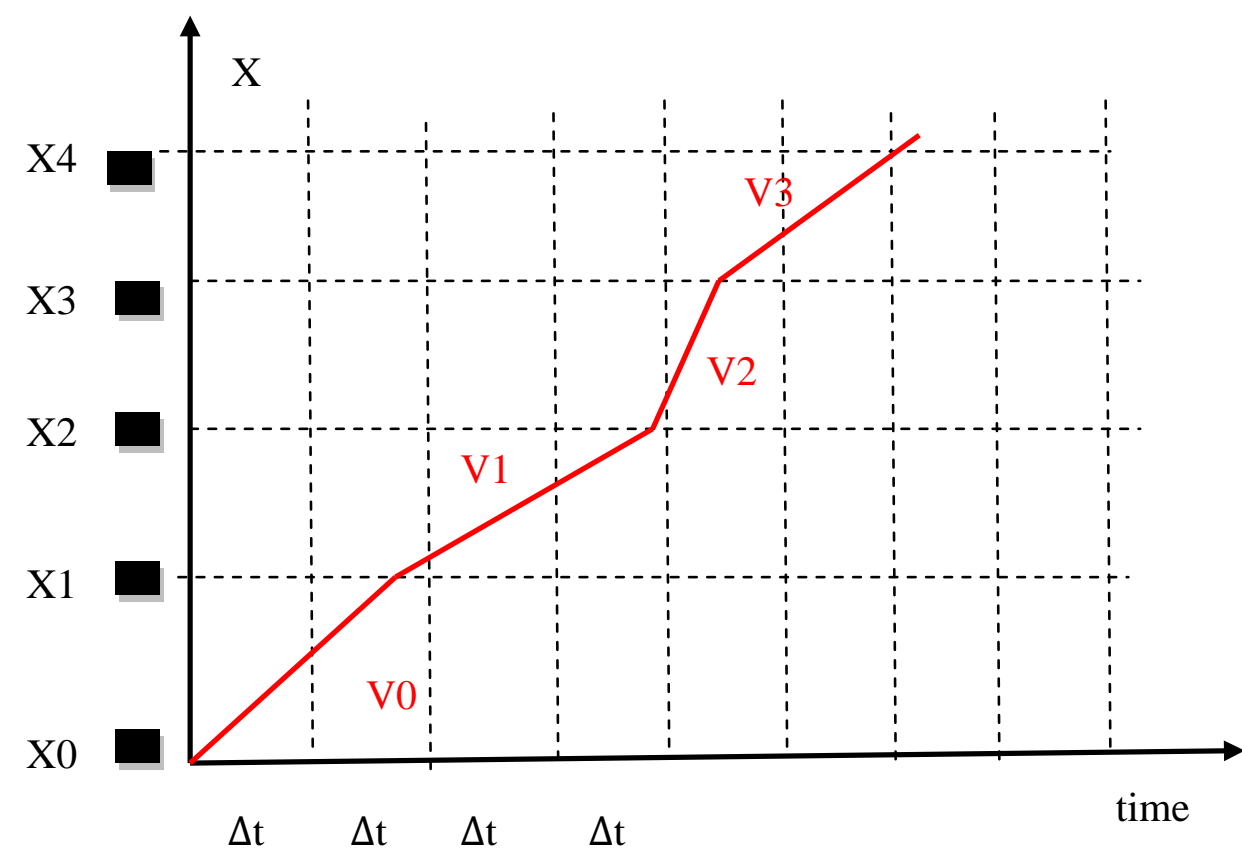

Figure 1. Algorithm for the Travel Time estimation

Congestion mapping consists of drawing of the iso-occupancy curves in space and time using the occupancy measurements of all installed loop detectors (space) and real data collection time slice (6 minutes).

\section{MEASUREMENT OF RELIABILITY}

A recent literature review on transport reliability (OECD/ITF, 2010) reveals a number of ways in which transport reliability has been defined and proposes another definition. According to that definition reliability is "the ability of the transport system to provide the expected level of service quality, on which users have organized their activities”. Indeed, a useful definition recognizes that network users time their actions according to expected network performance. The less predictable the network performance is, the greater the invehicle frustration; and greater the costs on personal and commercial schedules.

Measures of unreliability are important for identifying possible problems in the network performance.

When monitoring reliability, it is important to distinguish between the network operator perspective and user perspective. For the network operator, the focus is on network quality 
(what is provided and planned), while for the user, the focus is on how the variability of travel time is experienced.

Indeed, several definitions for travel time reliability exist and many different, but relevant, indicators have been proposed, with also several shortcomings. Here we use the same breakdown as presented in previous studies and divide these measures into four categories (Lomax et al., 2003 and Van Lint et al., 2004):

1. Statistical range methods.

2. Buffer time methods.

3. Tardy trip measures.

4. Probabilistic measures.

1 - Standard deviation (STD) and the coefficient of variation (COV) show the spread of the variability in travel time. They can be considered as cost-effective measures to monitor travel time variation and reliability, especially when variability is affected by a limited number of delays and when travel time distribution is not much skewed. Standard deviation is defined as:

$$
S T D=\sqrt{\frac{1}{N-1} \Sigma_{N}\left(T T_{i}-M\right)^{2}}
$$

while coefficient of variation is written as :

$$
\operatorname{COV}=\frac{S T D}{M}
$$


where $M$ denotes the mean travel time, $T T_{i}$ the $i^{\text {th }}$ travel time observation and $N$ the number of travel time observations.

A further consideration to use the standard deviation as a reliability indicator derives from recent studies that recommend defining travel time reliability as the standard deviation of travel time when incorporating reliability into cost-benefit assessment (Fosgerau et al., 2008). As a result, standard deviation is used to measure reliability in few countries where guidelines for cost-benefit assessment include reliability.

2 - The buffer methods are focusing on "the extra percentage of travel time due to travel time variability on a trip that a traveler should take into account in order to arrive on time”. These types of indices, especially the Buffer Index (BI) appear to relate particularly well to the way in which travelers make their decisions. It is defined as the ratio between the buffer time (BT) and the average travel time. Buffer time $(B T)$ is defined as the extra time a user has to add to the average travel time so as to arrive on time $95 \%$ of the time. It is computed as the difference between the 95th percentile travel time (TT95) and the mean travel time (M). Hence, Buffer Index is calculated as:

$$
B I=\frac{B T}{M}=\frac{T T_{95}-M}{M}
$$

The Buffer Index is useful in users' assessments of how much extra time has to be allowed for uncertainty in travel conditions. It hence answers simple questions such as "How much time do I need to allow?” or “When should I leave?”. For example, if the average travel time equals 20 minutes and the Buffer Index is $40 \%$, the buffer time equals $20 \times 0.40=8$ minutes. Therefore, to ensure on-time arrival with 95\% certainty, the traveler should allow 28 minutes for the normal trip of 20 minutes.

Planning Time (PT) is another concept often used. It gives the total time needed to plan for an on-time arrival $95 \%$ of the time as compared to free flow travel time. The Planning Time 
Index (PTI) is computed as the 95th percentile travel time ( $T T_{95}$ divided by free-flow travel time $\left(T T_{\text {free_flow }}\right)$

$$
P T I=\frac{T T_{95}}{T T_{\text {free flow }}}
$$

For example, if $P T I=1.60$ and $T T_{\text {fres flow }}=15$ minutes, a traveller should plan 24 minutes in total to ensure on-time arrival with 95\% certainty. As these indicators use the 95-percentile value of the travel time distribution as a reference for the definitions, they take into account more explicitly the extreme travel time delays.

3- Tardy trip measures indicate unreliability impacts using the amount of trips late. Indeed, if travelers only use the average trip time for their travel plans, they will be late to half their destinations and early to half (in round numbers). A Misery Index (MI) calculates the relative distance between mean travel time of the $20 \%$ most unlucky travelers and the mean travel time of all travelers. It is defined as

$$
M I=\frac{\left.M\right|_{T T_{i}>T T_{g 0}}-M}{M}
$$

where $T T_{80}$ is the $80^{\text {th }}$ percentile travel time.

4 - Probabilistic indicators $(\mathrm{Pr})$ calculate the probability that travel times occur within a specified interval of time. Probabilistic measures are parameterized in the sense that they use a threshold travel time, or a predefined time window, to differentiate between reliable and unreliable travel times. Probabilistic measures are useful to present policy goals, such as the Dutch target for reliability, according to which "at least 95\% of all travel time should not 
deviate more than 10 minutes from the median travel time” (Van Lint et al., 2008, Wakabayashi \& al, 2009). This can be presented by the following equation:

$$
\operatorname{Pr}\left(T T_{i} \geq \beta+T T_{50}\right)
$$

Equation (8) calculates the probability that travel times do not deviate by more than $\beta$ minutes

the median travel time. Parameter $\beta$ can be given any value. For example, $\beta=10$ minutes for routes less than $50 \mathrm{~km}$ in the Netherlands and is used in this paper.

\section{DESCRIPTIONS OF CANDIDATE STRATEGIES}

During the field assessment of ramp metering strategies, within the framework of the European Project EURAMP work plan (EURAMP, 2006), three adaptive control strategies were implemented: two local strategies, so called ALINEA and Variable Cycle ALINEA (VC_ALINEA), and a new coordinated strategy (CORDIN). In this paper, we omit VC_ALINEA results due the similarity with ALINEA. Consequently, the presented results are focused on ALINEA and CORDIN strategies.

\subsection{Isolated traffic responsive strategy: ALINEA}

ALINEA is based on a rigorous feedback philosophy (Bhouri et al., 1990; Haj-salem et al., 1990, 1991, 1995, Papageorgiou et al., 1989, 1991, Smulder et al., 1991). Since the main aim of ramp metering is to maintain the capacity flow downstream of the merging area, the control strategy for each controllable on-ramp should be based on downstream measurements. ALINEA, which was developed by the application of the well-known methods of the classical feedback theory, obtains the following form:

$$
r_{k}=r_{k-1}+K\left(O^{*}-O_{k}\right)
$$

where $r_{k}$ and $r_{k-1}$ are on-ramp volumes at discrete time periods $k$ and $k-1$ respectively, $O_{k}$ is the measured downstream occupancy at discrete time $k$ (the $O_{\text {out }}$ in Figure 2 ), $O^{*}$ is a pre-set 
desired occupancy value (typically $O^{*}$ is set around to the critical occupancy) and $K$ is a regulation parameter. The green light duration is computed as the ration between $\mathrm{r}_{k}$ and $\mathrm{Q}_{\mathrm{sat}}$ $\left(\mathrm{G}=\mathrm{r}_{\mathrm{k}} / Q_{\text {sat }}\right.$ ) where $\mathrm{Q}_{\mathrm{sat}}$ is the maximum observed on-ramp flow (typically Qsat $=0.5 \mathrm{vh} / \mathrm{s}$ ).
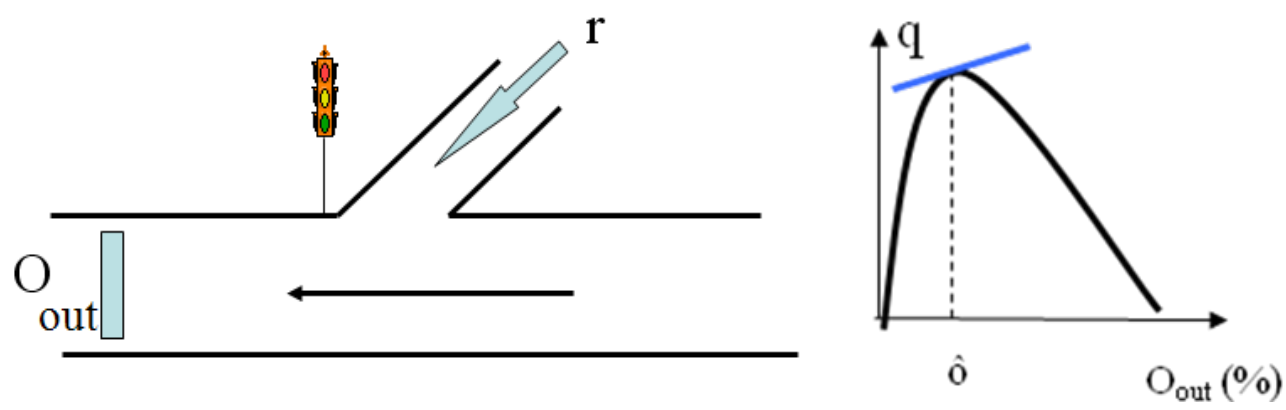

Figure 2. ALINEA description

\subsection{CORDIN: Coordinated Control Strategy}

The coordinated strategy is based on a heuristic approach. The main philosophy of this strategy consists of using free upstream on-ramp capacities in case of downstream motorway congestion. This philosophy is similar to the coordinated strategy HERO suggested by (Vong \& al. 2009, Papamichail \& al., 2010). However, the particularity of this strategy concerns the requirement of on-ramp measurement stations. In particular, no additive on-ramp measurement stations are needed and only the existing on-ramp measurement stations are used. This means that the on-ramp queue management is reduced to the released control constraint measurements only. The bloc diagram of the implemented coordinated strategy (CORDIN) is depicted in figure 3.

The following computation steps are applied:

1. Apply ALINEA to all controlled on-ramps, compute the ALINEA splits $\underline{U}_{a l}=G_{\text {ALINEA }} / C y c l e$ $\left(\underline{\mathrm{U}}_{\mathrm{al}} \leq 1, \underline{\mathrm{U}}_{\mathrm{al}}=1\right.$ means that traffic is fluid).

2. Find the location of the head of the congestion by testing if the first on-ramp $\left(r_{i}\right)$ where ALINEA is active $\left(\mathrm{U}_{\mathrm{al}}<1\right)$ and the queue constraint is not active.

3. For every Upstream on-ramp $r_{u p}=r_{i}+1, . .$, Nb_Ramps:

IF the queue constraint of the on-ramp $\left(\mathrm{r}_{\mathrm{up}}\right)$ is NOT active

THEN correct the ALINEA command according to: 
IF $\mathrm{r}_{\mathrm{up}}=\mathrm{r}_{\mathrm{i}}+1$ THEN $\mathrm{U}_{\text {coor }}=\alpha_{1} \mathrm{U}_{\mathrm{al}}$

ELSE $U_{\text {coor }}=\alpha_{2} U_{a l}$ for the other upstream ramps,

where $\left(\mathrm{V}_{1}\right)$ and $\left(\mathrm{V}_{2}\right)$ are parameters to be calibrated;

OTHERWISE do nothing.

4. Apply the new coordinated control sets on the field.

5. Wait until the next cycle time and Go to step 1.

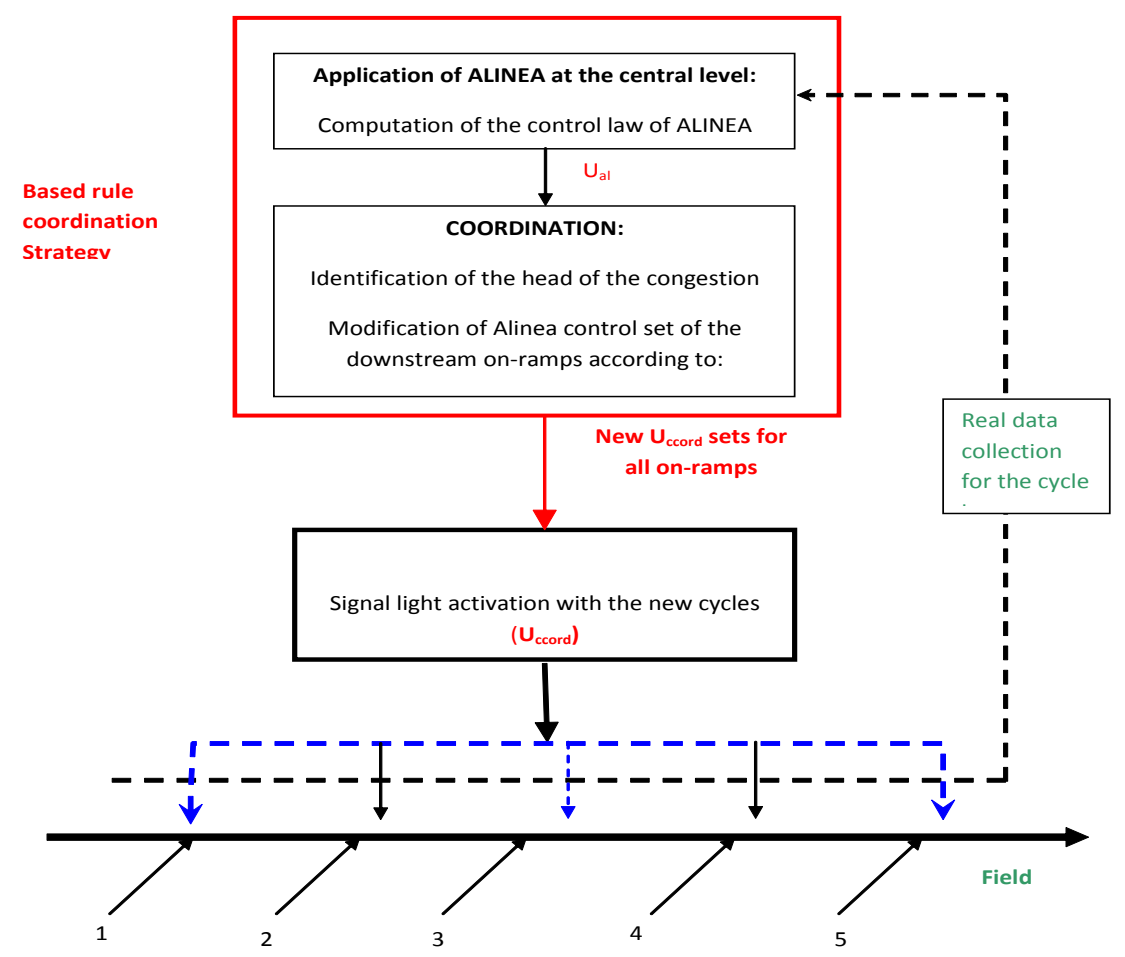

Figure 3. Bloc diagram of CORDIN strategy

For each control strategy, the characteristics of the cycle time are the following: cycle duration $=40 \mathrm{Sec}$, Max_green= $30 \mathrm{sec}$, Min_green $=10 \mathrm{sec}$, Amber= $5 \mathrm{sec}$. These durations have been found in previous field trials as an optimal solution (EURAMP, 2007).

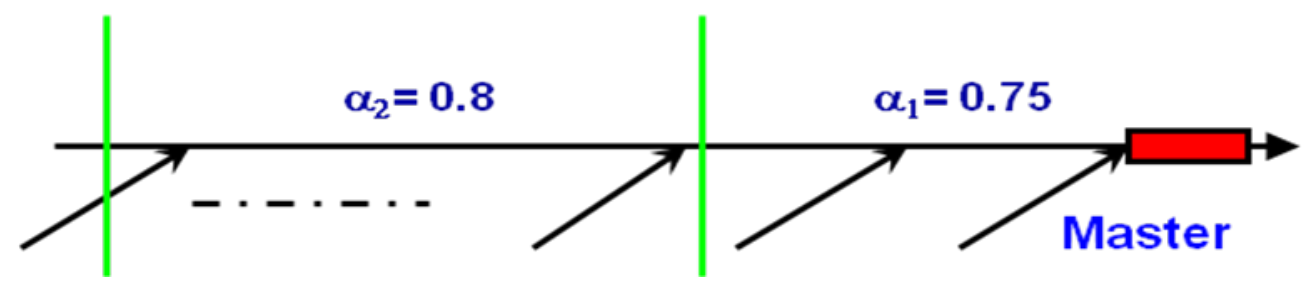


Figure 4. Example of CORDIN parameters

Based on the cycle characteristics, the $\left(\alpha_{1}, \alpha_{2}\right)$ CORDIN parameters are fixed as follows: $\alpha_{1}=$ Max_green $/$ Cycle $=30 / 40=0.75$ and $\alpha_{2}=0.80$. The $\alpha_{2}$ parameter is fixed arbitrary in order to less penalize the user of far upstream on-ramps. Figure 4 depicts one example of the applied correction parameters $\left(\alpha_{1}, \alpha_{2}\right)$ after the detection of the congestion head (MASTER on-ramp).

\section{TEST SITE AND AVAILBLE DATA DESCRIPTION}

The reported field trials have been conducted within the framework of the European Project EURAMP work plan. They have been designed and carried out on the France test site aiming at the traffic impact assessment of coordinated and isolated ramp metering strategies. Field trials include a comprehensive data collection from the considered network (A6W motorway) over several months with isolated and coordinated ramp metering strategies (EURAMP, 2006).

The motorway section (A6W) of the French field test (see Figure 5) comprises 5 on-ramps (Chilly-Mazarin, Savigny, Viry, Grigny and Ris-Orangis) which are fully equipped with signal lights and loop measurement stations. The total motorway length is around $20 \mathrm{~km}$. This motorway section is fully equipped with traffic flow, occupancy rate and speed measurement stations roughly every 500 meters. The controlled ramps include two measurement stations each. The upstream one is used to detect surface intersection blocking, and the downstream one is used by the on-ramp metering strategy. The considered test site is the most critical area of the A6W motorway toward Paris. Morning and evening peak congestions are observed over several hours and several kilometers. 


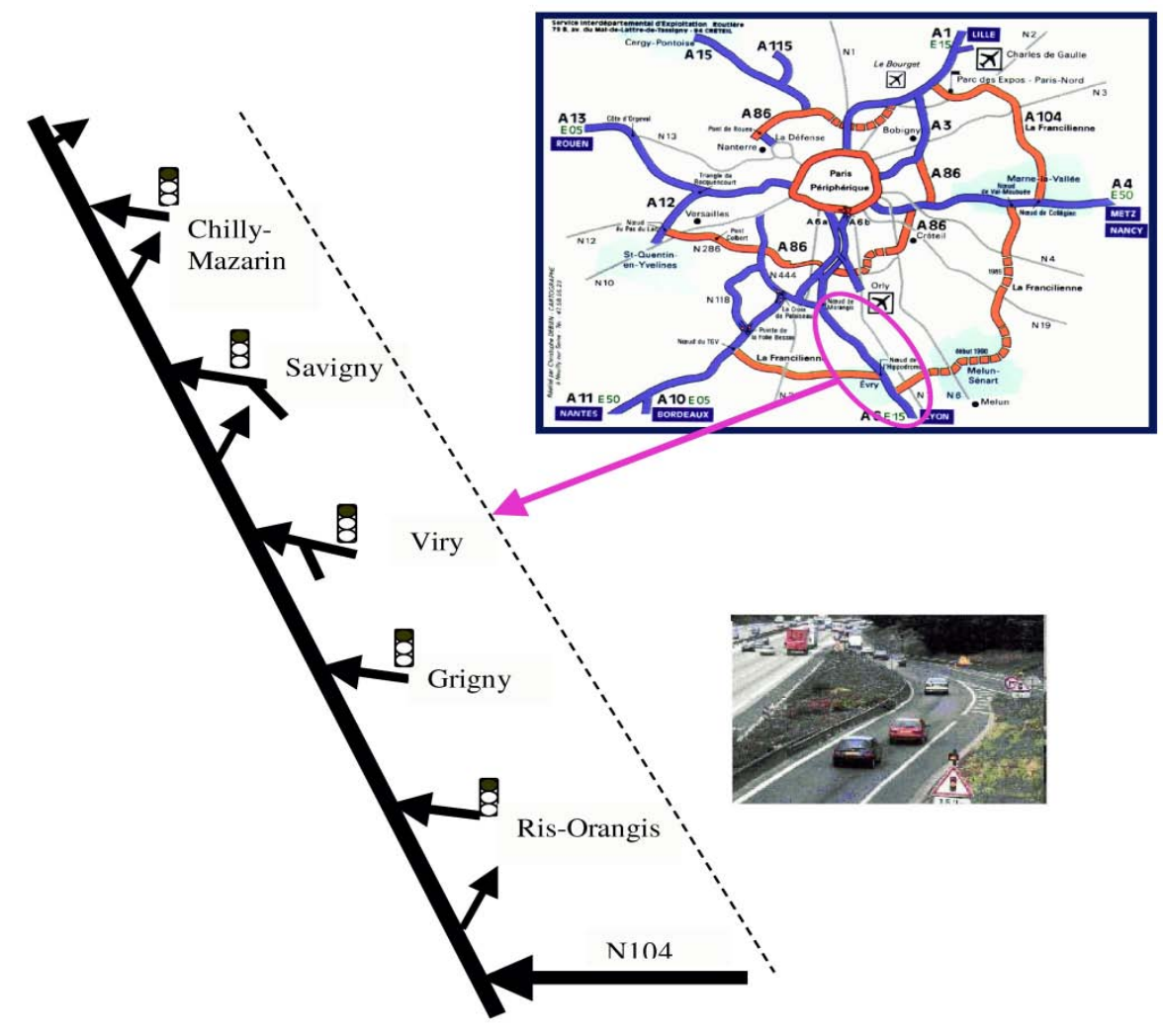

Figure 5. A6W Field test site.

The candidate control strategies were applied over alternate weeks over the period from the middle of September 2005 until the end of January 2007, after which comparative assessments of the traffic impacts were performed.

Full 140 days of collected data were extracted from the database of the traffic management system. Screening the collected data was firstly necessary in order to discard days which include major detector failures. Secondly, all days with atypical traffic patterns (essentially weekends and holidays) were discarded. Finally - and in order to preserve comparability - all days that involved significant incidents or accidents (according to the incident files provided by the police), were also left out. This screening procedure eventually delivered data for 11 , 10 and 9 days for No control, ALINEA and CORDIN strategies respectively. In order to 
minimize the impact of demand variations on the comparative process, the selected days were averaged for each strategy.

\section{EVALUATION RESULTS}

\subsection{Traffic Impact Results}

Table 1 reports results of the selected traffic indices of the overall network (carriage way and on-ramps) during the time period of 6h-11h. Compared to the No control case, the CORDIN strategy presents a better performance than ALINEA. As a matter of fact, with same Total Travel Distance (TTD) than the No control case, the Total Time Spent (TTS) index is reduced by $12 \%$. We can underline that the time period considered covers a fluid traffic condition at the beginning (6:00-6:30am) and at the end (10:00-11:00am) of the period. Investigation between 8:30 and 9:30 where the ramp metering strategies are continuously active indicated that all traffic indices are improved. Compared to the No control case, these improvements of the TTS were $12 \%$ and $21 \%$ and for the TTD $+0.1 \%$ and $+2 \%$ for ALINEA and CORDIN respectively.

\begin{tabular}{|c|c|c|c|c|c|}
\cline { 2 - 6 } \multicolumn{1}{c|}{} & NC & ALINEA & CORDIN & ALINEA & CORDIN \\
\hline TTS (vh*h) & 1399 & 1263 & 1231 & $-9.8 \%$ & $-12.0 \%$ \\
\hline TTD (vh*km) & 6266 & 6141 & 6260 & $-2.0 \%$ & $-0.0 \%$ \\
\hline MS (km/h) & 51.7 & 53.9 & 57.3 & $4.3 \%$ & $10.9 \%$ \\
\hline
\end{tabular}

Table 1. Motorway Traffic impact indices (6h-11h) 
Figure 6 depicts the congestion mapping of A6W motorway. This figure represents the isooccupancy curves in space and time for all candidate strategies: No Control, ALINEA and CORDIN. The different colors represent the level of the congestion (eg: green $-0<$ occ $<$ $11 \%$ : fluid, yellow: dense $-11<$ occ $<21 \%$ ). We can observe that the head of the congestion is located at Savigny on-ramp at around $6 \mathrm{~h} 30$ am and spill back to the downstream Viry onramp and Grigny. The qualitative assessments of the congestion area are in favor of CORDIN and confirm the quantitative results of the TTS indices.
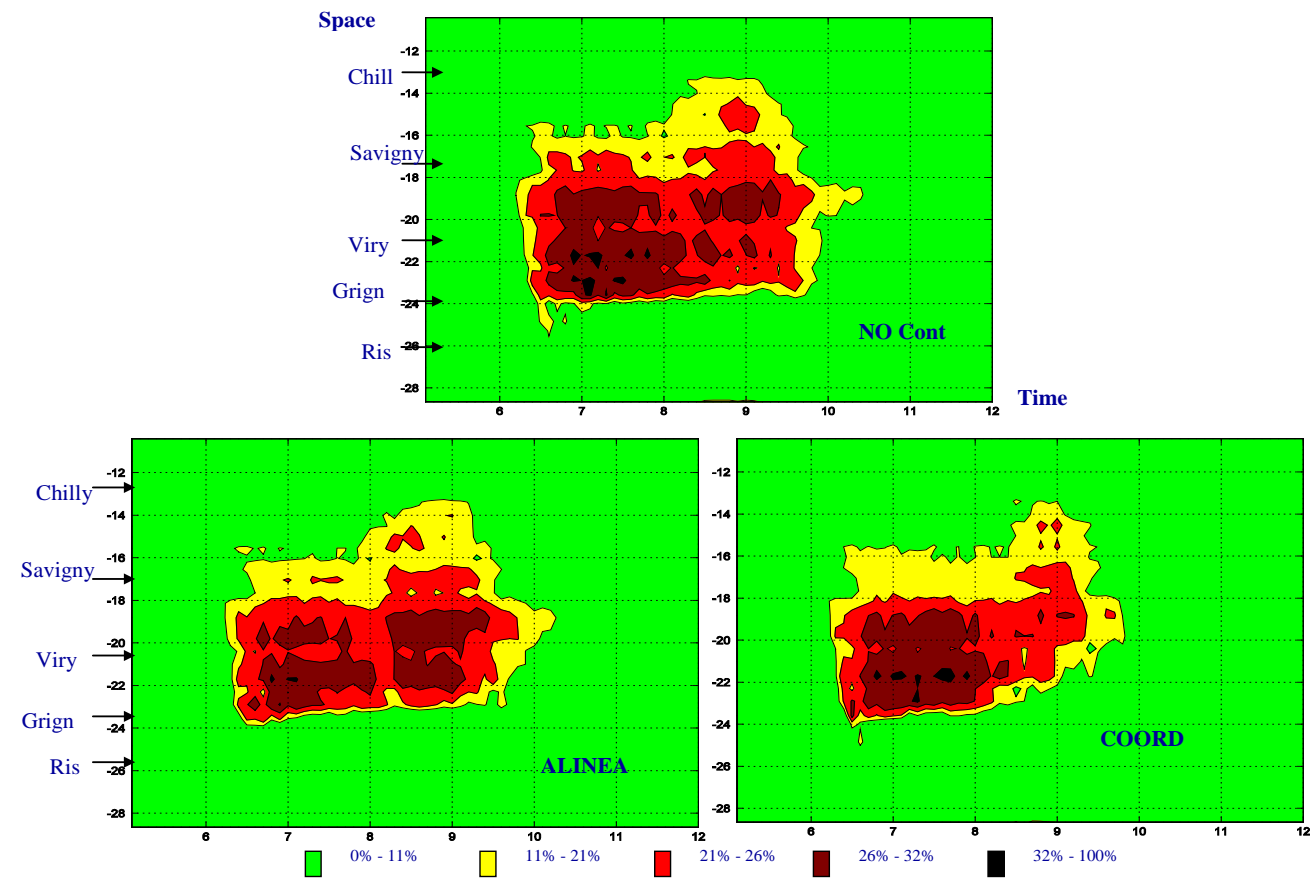

Figure 6. Congestion mapping of the 3 strategies

With respect to the Total Travel Time, Figure 7 depicts the gain evolution in space in term of the mean travel time index. The space is subdivided in five points and corresponding to the on-ramps locations. The CORDIN strategy gives better results than the isolated strategies. As far as the travelled distance increases, the gain in term of mean travel times increase also. The maximum gain of $17 \%$ is observed for the CORDIN strategy. 


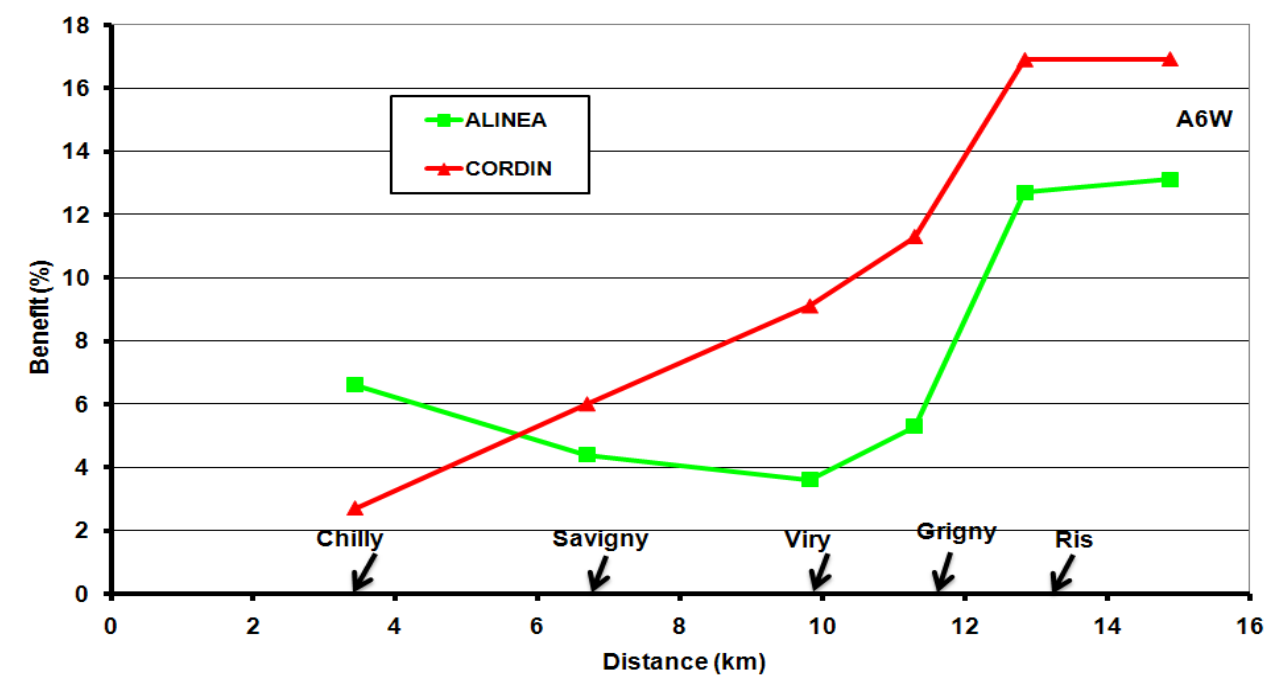

Figure 7. Mean Travel Time Benefit (\%) of the candidate strategies by on-ramps

The above results on traffic impact are in favor of the implementation of coordinated ramp metering strategies. Traffic conditions can be significantly improved with less effort in terms of measurement installations, compared with the isolated ramp metering strategies.

In the next section we turn our focus on the evaluation of reliability. While for measuring the traffic impact we used the mean travel time overall on the selected days, for the measurement of reliability, the evaluation is based on the predicted travel day by day in order to point out the variability.

\subsection{Travel time variability}

In this section, we study the impacts of ramp metering strategies on travel time variability. The overall results can be seen in Figure 8, which compares daily travel time variability between the three tested strategies No-Control, ALINEA and CORDIN respectively (Bhouri and Haj-Salem, 2009). First observation is that, in addition to the improvement of the average time, the travel time variability is reduced by using ramp metering control strategies. Secondly, there are no significant differences on the daily travel time variability between ALINEA and CORDIN strategy. 


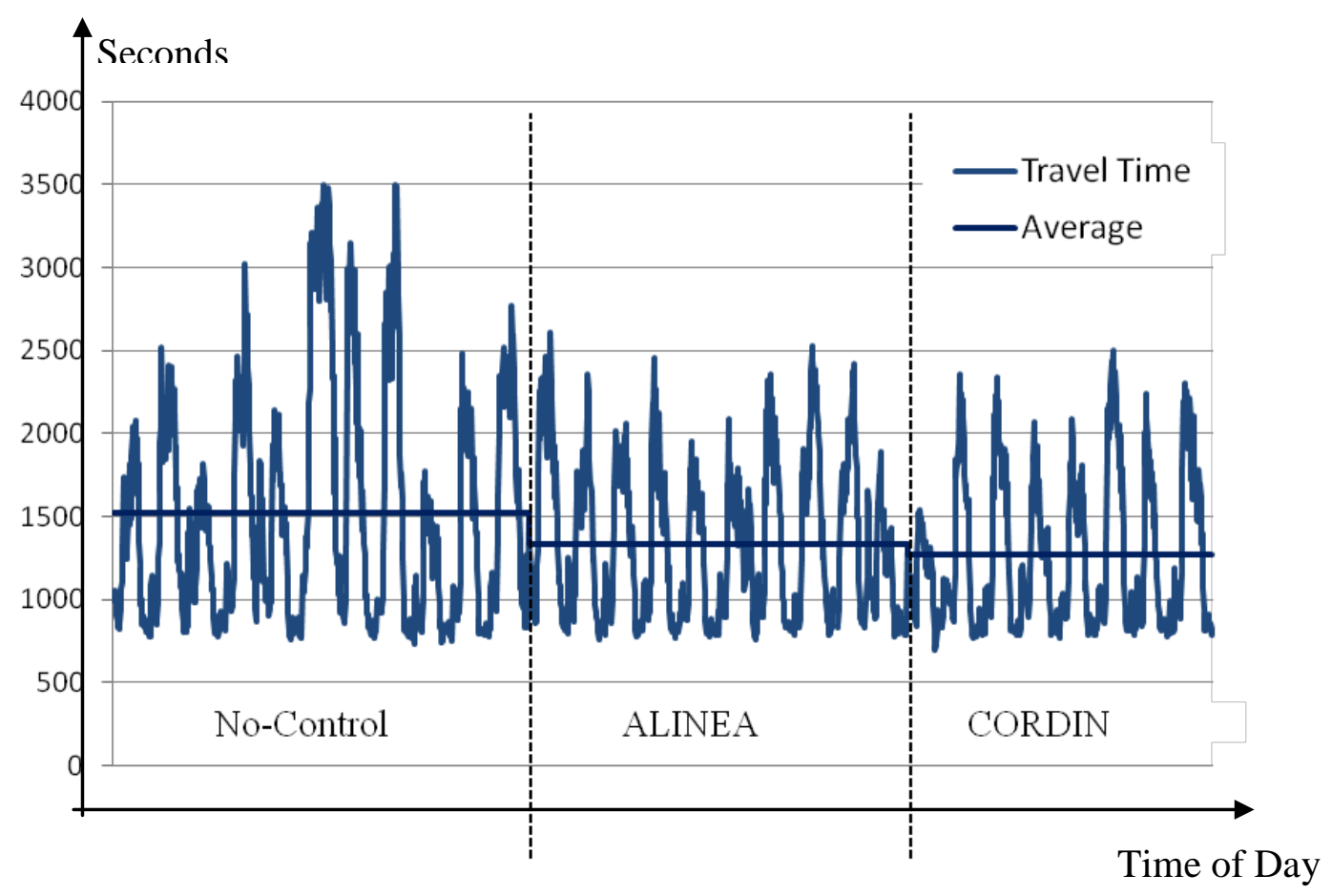

Figure 8. Comparison of results between the three strategies

Table 2 includes the travel time variability for the two tested ramp metering strategies: ALINEA and CORDIN with a reference to No Control case. Applying ramp metering strategy at the five on-ramps of A6W motorway improves reliability by $24-37 \%$, depending on the indicator used. Results are consistent in the direction of change with, however, variation in the size of the impact. The wider the travel time distribution, the less reliable travel times are. As indicated in Table 2, overall the spread or variation (STD or COV) of the travel time distribution becomes smaller (more reliable) when applying ramp metering. The Misery Index (MI) indicates that $20 \%$ of the most unlucky travelers experienced a travel time $76 \%$ worse than the average travel time in case of the No-Control. The index was reduced to 53\% and $58 \%$ in case of ALINEA and CORDIN respectively. Probability index $(\mathrm{Pr})$ shows that, without active management, $28 \%$ of users experience more than 10 minutes of delay as compared with the median travel time. Again, ramp metering reduced this to only $18 \%$ of users when ALINEA was applied. 


\begin{tabular}{|l|l|c|c|c|c|c|}
\hline \multicolumn{1}{|c|}{ Category } & \multicolumn{1}{|c|}{ Acronym } & No & Alinea & Cordin & Gain & Gain \\
\hline Statistical & STD (sec) & 706 & 463 & 456 & $34 \%$ & $35 \%$ \\
\hline & COV & $46 \%$ & $35 \%$ & $36 \%$ & $25 \%$ & $22 \%$ \\
\hline Tardy Trip & MI & $76 \%$ & $53 \%$ & $58 \%$ & $31 \%$ & $24 \%$ \\
\hline Probabilistic & Pr & 0,28 & 0,18 & 0,21 & $35 \%$ & $24 \%$ \\
\hline Buffer Index & BI & & & & & $32 \%$ \\
\hline & PTI & $98 \%$ & $62 \%$ & $67 \%$ & $37 \%$ & $30 \%$ \\
\hline
\end{tabular}

Table 2. Statistical indicators

While the results between the coordinated strategy and ALINEA are rather similar, the specific characteristics of each measure results in variations of findings between different indicators. For the policy maker, the variation in findings presented above can be problematic. We can only draw an overall conclusion of that ramp metering seems to reduce variability in general. The results are also difficult to communicate to users of the network who are more accustomed to making decisions based on time (minutes), rather than on percentages.

In the following, obtained results on the average Travel Time (TT) are discussed based on the Buffer Time (BT) and Planning Time (PT), in minutes. Table 3 shows that a user who plans to arrive at his destination on time, with 95\% certainty, during the morning peak period on the A6W has to take into account the mean travel time of 25 minutes, and add another 25 minutes as a "buffer" to ensure ontime arrival (in No Control case). Hence the actual travel time during the morning peak is doubled due to uncertainty and variability in travel time.

However, when introducing active management through ramp metering (ALINEA or CORDIN), user's planning time is reduced by 14 minutes, with the total time needed for the trip declining from 
50 to 36 minutes. The main improvement from the user perspective comes, indeed, from reduced variability in travel time while the mean travel time only improves by 3-4 minutes. For the average travel time, the improvement is slightly greater with the coordinated strategy than ALINEA. However, from a reliability point of view, the observed gain of ALINEA and CORDIN strategies are similar.

\begin{tabular}{|c|c|c|c|c|c|c|}
\hline & $\begin{array}{c}\text { TT } \\
(\mathrm{min})\end{array}$ & Gain (\%) & $\begin{array}{c}\text { BT } \\
(\mathrm{min})\end{array}$ & Gain (\%) & $\begin{array}{c}\text { PT } \\
(\mathrm{min})\end{array}$ & Gain (\%) \\
\hline No-Control & 25.4 & $* * *$ & 25 & $* * *$ & 50,4 & $* * *$ \\
\hline ALINEA & 22.3 & 12 & 13.8 & 44.5 & 36.2 & 28 \\
\hline CORDIN & 21.2 & 17 & 14.1 & 43 & 35.3 & 30 \\
\hline
\end{tabular}

Table 3. Travel time, buffer time and planning time

The above data are illustrated in Figure 9 showing the spread of the travel time for the NoControl case, ALINEA and CORDIN. The figure clearly shows how the tail of the travel time distribution is shortened and the buffer time is reduced when ramp metering is in use. Figure $9 \mathrm{~b}$ also illustrates that there are no significant differences between coordinated strategy and ALINEA.

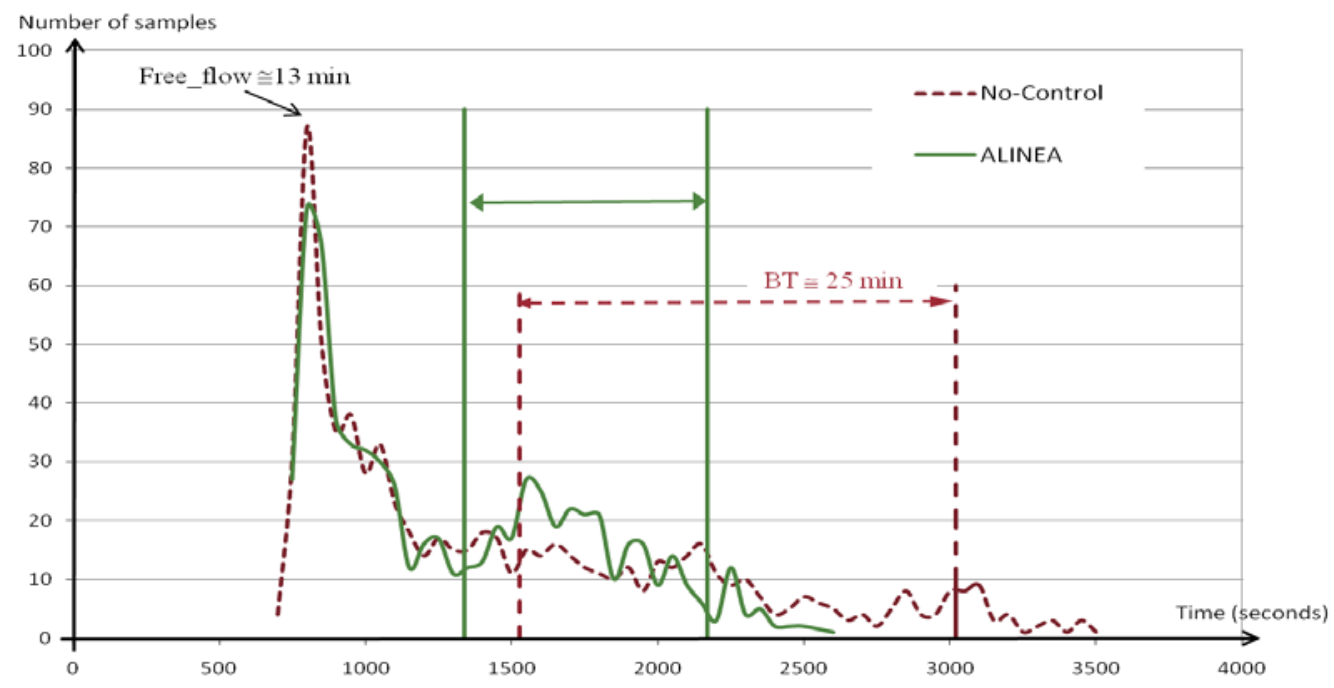


Figure 9(a). Reliability indicators for ALINEA and No-control cases

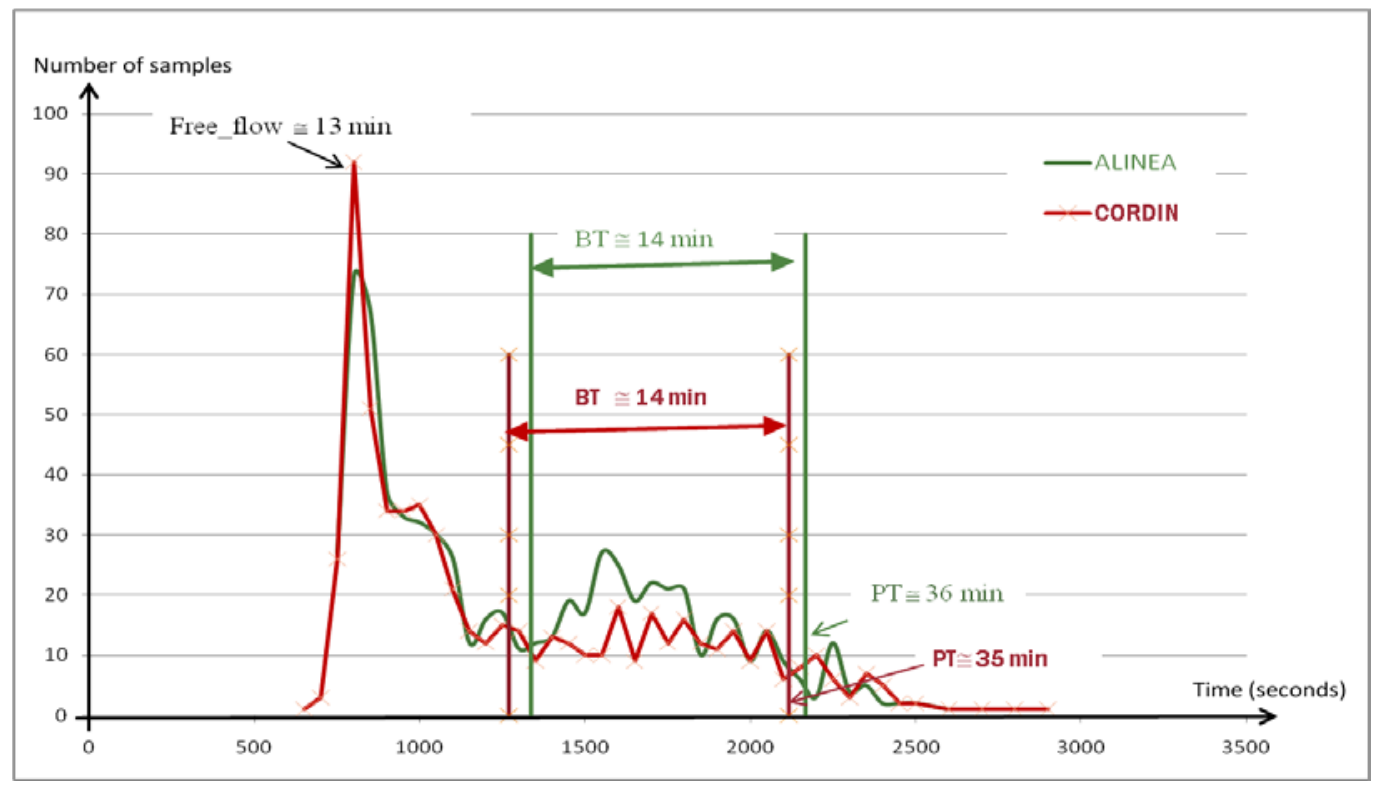

Figure 9(b). Reliability indicators for ALINEA and CORDIN cases

Figure 10 depicts the difference between congestion and reliability by time of day. As the morning peak starts at around $6 \mathrm{am}$, travel time increases sharply from around 17 minutes to over 35 minutes by 6:42 am. It remains at this level until 9 am, starting then slowly to decrease until, at around $10 \mathrm{am}$, it has reached almost the pre-peak levels. At the congestion onset, the unreliability of travel time also increases rapidly, and the buffer time grows from 4 minutes at around 6 am to 14 minutes by 6:42 am. However, in contrast to travel time, the buffer time continues to increase all the way until 10am, finally reaching nearly 22 minutes. This may be explained by the fact that, during peak congestion, travel is consistently slow; whereas as congestion dissolves travelers are faced with more variable speeds affecting travel time distribution, including extreme observations at the tail end of the distribution. 


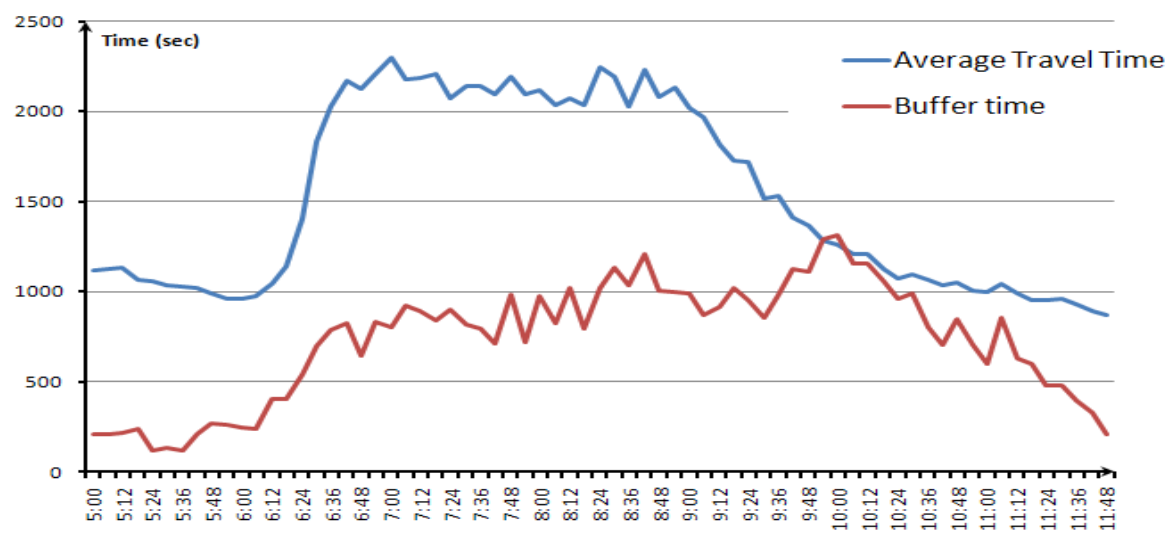

Figure 10. Congestion and reliability

Finally, a comparison of the travel time reliability given by the three strategies (Figure 11) shows a gain in the intensity and the duration of the perturbation when a ramp metering strategy is applied (ALINEA or CORDIN). At 10:30am, a traveller needs to add nearly 2.6 times more time, compared with the free flow travel time, to ensure on-time arrival in $95 \%$ when no ramp metering is in use. In comparison, a traveller needs to add only 1.25 times the free flow travel time at the same time when the coordinated strategy is applied. Although we observe small differences between the two strategies, they do not seem to be significant.

To summarise, the results on both traffic and travel time reliability evaluations suggest that ramp metering strategies impose a significant improvement in traffic conditions. 


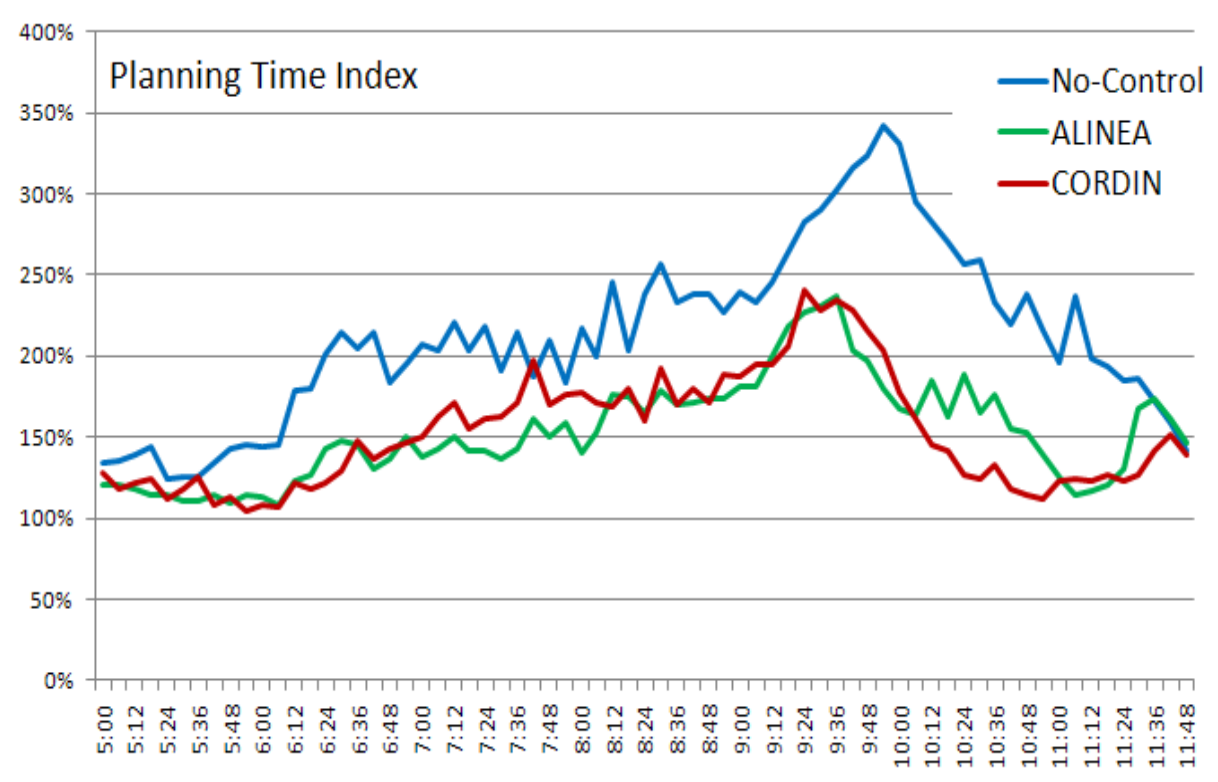

Figure 11. Comparison of Planning Time Index for the three strategies

\section{CONCLUSIONS}

In this paper we have conducted an analysis of the performance of both isolated and coordinated ramp metering strategies using real data measurements. In particular, in addition to the traditional assessment of the traffic impact we also assess and the impact of ramp metering on travel time reliability. Our results suggest that travel time reliability can be incorporated into assessment without any significant difficulties providing additional indication of the effectiveness of different measures for traffic management authorities.

Reliability of travel time is increasingly recognized as an important factor to be accounted for in an assessment. Indeed, we find that applying ramp metering on a French motorway A6W significantly improves, not only the average travel time, but the reliability of travel times and hence reduces the time needed for a traveler to ensure on-time arrival. While the average travel time is improved by 3 minutes, the buffer time is reduced by 11-12 minutes.

Furthermore, the obtained results indicate that the coordinated strategy is more efficient in terms of total time spent on the motorway than the isolated strategy. With respect to the reliability, there are no significant differences between the two strategies on the main 
motorway axis. Compared to the no control case, both traffic responsive strategies reduce the variability in travel time by $24-37 \%$ depending on the measure used.

\section{REFERENCES}

Bhouri, N., Kauppila, J. (2011) “Managing Highways for Better Reliability - Assessing Reliability Benefits of Ramp Metering, Transportation Research Record, Journal of the Transportation Research Board, No. 2229, Transportation Research Board of the National Academies, Washington, D.C., 2011, pp. 1-7.

Bhouri, N. , H. Haj-Salem, (2009) “Motorway Ramp Metering as a tool for improving the travel time reliability”, 13th European Working Group in Transportation Conference (EWGT), Padua, Italy, 23-25 September 2009.

Bhouri N., Papageorgiou M., Blosseville JM. (1990) "Optimal control of traffic flow on periurban ring ways with an application to the Boulevard Périphérique in Paris" IFAC, 11th World Congress, Tallinn, URSS.

Chen, A., H. Yang, H.K. Lo and W.H. Tang (1999) A capacity related reliability for transportation networks. Journal of Advanced Transportation, 2 (vol. 33) pp. 183-200.

DACCORD, Deliverable D10.3, (1999), “Final report of assessment results”, European Telematics Applications Programme (TAP), project TR 1007, February 1999.

EURAMP, European Project IST-2002-23110 (2007),"Evaluation Results report”, Deliverable D6.3, European Commission, July 2007. 
EURAMP, European Project IST-2002-23110 (2006),”Simulation testing for local ramp metering strategies”, Deliverable D3.2, European Commission, February 2006.

FHWA (2006), “Travel time reliability: Making it there on time, all the time”; http://www.ops.fhwa.dot.gov/publications/tt_reliability/TTR_Report.htm.

Fosgerau, M., Hjorth, K, Brems, C. and Fukuda D. (2008), Travel time variability Definition and valuation. Danish Department of Transport 1:2008.

Haj-Salem, H., Blosseville, J.-M., Papageorgiou, (1990) "ALINEA - A Local Feedback Control Law for on-ramp metering: A real life study". 3rd IEE Intern. Conf. on Road Traffic Control, London, U.K., pp.194-198.

Haj-Salem, H., Papageorgiou, M. (1995) "Ramp Metering Impact on Urban Corridor Traffic : Field Results". Transportation Research -A._Vol 29A, No.4, pp 303-319.

Haj-Salem, H., Papageorgiou, M. (1991) "Linear Motorway Control: Real life Study in France", DRIVE-1 CHRISTIANE (V1035), Deliv. $N^{\circ}$ 13. INRETS, France.

H. Haj Salem, M. Boukhnifer, Mabrouk H. S. Mammar (2010), "Le Contrôle d'accès généralisé sur les autoroutes de l'île de France : Études en Simulation et sur site réel ", MOSIM’2010, 8ème ENIM IFAC Conférence Internationale de Modélisation et Simulation, Évaluation et Optimisation des Systèmes Innovants, Hammamet, Tunisie. 
H. Haj-Salem, C. Ramananjoana, J.P Lebacque (2006), “Risk Index Model for Real-Time Prediction of Potential traffic Crash on Urban Motorway”. Proceedings of the EWGT2006, Advances in Traffic and transportation Systems Analysis, BARI, Italy.

Lomax, T., Schrank, D., Turner, S., Margiotta, R., (2003), "Selecting travel reliability measures”, Texas transportation institute Cambridge systematics, Inc.

McKinnon A., J. Edwards, M. Piecyk and A. Palmer (2009), “Traffic Congestion, Reliability and Logistical Performance: A Multi-Sectoral Assessment”. International Journal of Logistics Research and Applications, Volume 12, Issue 5 , pp 331 - 345

OECD/ITF (2010) Improving Reliability on Surface Transport Networks. OECD, Paris, 2010.

OECD/ECMT (2007), Managing Urban Traffic Congestion. OECD, Paris, 2007.

Papamichail I., Papageorgiou M., Vong V., and Gaffney J., 2010. “HERO coordinated ramp metering implemented at the Monash Freeway, Australia”. Transportation Research Record: Journal of the Transportation Research Board. 2178, pp. 10-20, 2010

Papageorgiou, M., Haj-Salem, H. Blosseville, J.-M. (1991) "ALINEA: A Local Feedback Control Law for on-ramp metering". Transportation Research Record 1320, pp. 58-64.

Smulders, S., Middelham, F. (1991)."Isolated ramp metering: real life study in The Netherlands". DRIVE-1 CHRISTIANE (V1035), Deliv. $N^{\circ} 7 a$, Rijkwaterstaat, Rotterdam, Netherlands. 
Papageorgiou, M., Haj-Salem, H. Blosseville, J.-M., Bhouri, N. (1989) " Modelling and real time control of traffic flow on the Boulevard Périphérique in Paris ". IFAC/IFIP/IFORS International Symposium CCCT'89, septembre, Paris.

Van Lint, J W C, Van der Zijpp, N J, (2003). “Improving Travel-time Estimation Algorithm by Using Dual Loop Detectors”, Transportation Research Record, Issue Number: 1855, p. 41-48

Van Lint, J.W.C.,(2004) "Freeway Travel Time Reliability Maps; Using the shape of the dayto-day travel time distribution”, Paper presented at the 8th TRAIL congress, 2004 November 23, Rotterdam, The Netherlands.

Van Lint, J.W.C., van Zuylen, H. J., Tu, H. (2008 ) “Travel Time unreliability on freeways: Why measures based on variance tell only half the story". Transportation Research Part A, $\mathrm{N}^{\circ} 42$, , pp 258-277.

Wakabayashi, H., Matsumot, Y. and Suzuki, A. ( 2009 ) "Comparative study on travel time reliability indexes for highway users and administrations”. EWGT’09. Padova, Italy.

Vong V. and Gaffney J., (2009). "Implementing Traffic Management Tools to Mitigate Freeway Congestion”, In Proc. 2nd TRB International Symposium on Freeway and Tollway Operations, Honolulu, Hawaii. 